\title{
Thermophysical Characteristics of OSIRIS-REx Target Asteroid (101955) Bennu
}

\author{
Liangliang $\mathbf{Y u}^{1,2}$ and Jianghui $\mathbf{J i}^{1}$ \\ ${ }^{1}$ Key Laboratory of Planetary Sciences, Purple Mountain Observatory, Chinese Academy of \\ Sciences, Nanjing 210008, China \\ ${ }^{2}$ Lunar and Planetary Science Laboratory, Macau University of Science and Technology, \\ Taipa, Macau \\ email: jijh@pmo.ac.cn, yullmoon@pmo.ac.cn
}

\begin{abstract}
In this work, we investigate the thermophysical properties, including thermal inertia, roughness fraction and surface grain size of OSIRIS-REx target asteroid (101955) Bennu by using a thermophysical model with the recently updated 3D radar-derived shape model (Nolan et al., 2013) and mid-infrared observations (Müller et al. 2012, Emery et al., 2014). We find that the asteroid bears an effective diameter of $510_{-40}^{+6} \mathrm{~m}$, a geometric albedo of $0.047_{-0.0011}^{+0.0083}$, a roughness fraction of $0.04_{-0.04}^{+0.26}$, and thermal inertia of $240_{-60}^{+440} \mathrm{Jm}^{-2} \mathrm{~s}^{-0.5} \mathrm{~K}^{-1}$ for our best-fit solution. The best-estimate thermal inertia suggests that fine-grained regolith may cover a large portion of Bennu's surface, where a grain size may vary from 1.3 to $31 \mathrm{~mm}$. Our outcome suggests that Bennu is suitable for the OSIRIS-REx mission to return samples to Earth.
\end{abstract}

Keywords. radiation mechanisms: thermal, minor planets, asteroids: individual: (101955) Bennu, infrared: general

\section{Introduction}

(101955) Bennu is an Apollo-type near-Earth asteroid (NEA) given its orbital characteristics. Bennu is a potential Earth impactor with a relatively high impact probability of approximately $3.7 \times 10^{-4}$ (Milani et al., 2009, Chesley et al., 2014). Recently, Chesley et al. (2014) showed that the semimajor axis of Bennu drifts at an averaged rate $d a / d t=(-19.0 \pm 0.1) \times 10^{-4} \mathrm{au} \cdot \mathrm{Myr}^{-1}$ due to the Yarkovsky effect, and further predicted dozens of potential impacts for this asteroid from 2175 to 2196. Since its orbit makes it especially accessible for the spacecraft, Bennu is considered as one of the potentially hazardous asteroids (PHA) and was chosen as a suitable target for NASA's OSIRIS-REx sample return mission (Lauretta et al., 2012). The OSIRIS-REx spacecraft is scheduled to be launched in 2016 .

Several researchers investigated the thermophysical features of Bennu. Müller et al. (2012) derived Bennu's thermal inertia of $\sim 650 \mathrm{Jm}^{-2} \mathrm{~s}^{-0.5} \mathrm{~K}^{-1}$ with thermophysical model, based on observations from Herschel/PACS, ESO-VISIR, Spitzer-IRS and SpitzerPUI. Recently, Emery et al., (2014) showed an update thermal inertia of Bennu approximately $310 \pm 70 \mathrm{Jm}^{-2} \mathrm{~s}^{-0.5} \mathrm{~K}^{-1}$ from a thermophysical analysis of Spitzer-IRS spectra and a multi-band thermal lightcurve. There are two main different aspects between the work of (Emery et al., 2014) and (Müller et al. 2012): first, the former considered a 3D radar-derived shape model by (Nolan et al., 2013) in the modeling process, whereas the latter adopted a simple spherical shape model; second, the mid-infrared observations they used were not idenfical, in that IRAC and IRS peak-up data were included in Emery et al., (2014), but not utilized in (Müller et al. 2012). 
In this paper, we adopt independently developed thermophysical simulation codes from (Yu, Ji \& Wang 2014) based on the Advanced Thermal Physical Model (ATPM) (Rozitis \& Green 2011), to investigate the surface thermophysical characteristics of Bennu. In our modelling process, we utilize Bennu's radar-derived shape model given by (Nolan et al., 2013) rather than a spherical approximation shape (Müller et al. 2012). Moreover, we added the mid-infrared data gathered from four groups of observations, at various phase angles, by Spitzer-PUI, Spitzer-IRAC, Herschel/PACS and ESO VLT/VISIR (Müller et al. 2012, Emery et al., 2014). By fitting all observations, we obtain a thermal inertia of Bennu that is slightly lower than that of (Emery et al., 2014), which indicates an important evidence of the fine-grained regolith on Bennu's surface (for details see Yu \& Ji 2015). Moreover, using the derived thermal inertia, we estimate the grain size of the regolith from a thermal conductivity model of (Gundlach \& Blum 2013).

\section{Analysis}

Figure 1 shows a contour of $\chi^{2}$ in the 2-dimensional parameter space $\left(\Gamma, f_{R}\right)$, where the increase of $\chi^{2}$ is shown by color bar from blue to red. The black '+' corresponds to the minimum $\chi_{\min }^{2}$. And in this case, we have $\Gamma=240 \mathrm{Jm}^{-2} \mathrm{~s}^{-0.5} \mathrm{~K}^{-1}$ and $f_{\mathrm{R}}=0.04$, as best fit solutions to the observations. The blue curve is the profile of $\chi_{\min }^{2}+1$, which is assumed as the $1 \sigma$ limit of the free fit parameters $\Gamma$ and $f_{\mathrm{R}}$. The blue profile forms a closed region in the $\left(\Gamma, f_{\mathrm{R}}\right)$ space. Thus, we can simultaneously constrain thermal inertia and roughness fraction in 2-dimensional space, considering the $1 \sigma$ limit. However, the contour curves of $\chi^{2}$ above $1 \sigma$ cannot form closed regions, suggesting that the degeneracy of thermal inertia and roughness fraction cannot be removed so well like the $1 \sigma$ level, thus thermal inertia and roughness fraction may be simply separated at the $1 \sigma$ level based on the ATPM calculations. Therefore, if the $1 \sigma$ limit is reliable, we may safely conclude that the roughness fraction is possible in the range of $0 \sim 0.3$, and the thermal inertia may be in the range of $180 \sim 680 \mathrm{Jm}^{-2} \mathrm{~s}^{-0.5} \mathrm{~K}^{-1}$. Our results agree with both earlier investigations of (Müller et al. 2012) and (Emery et al., 2014).

With the above derived thermal inertia, the grain size of Bennu can be estimated according to the method of (Gundlach \& Blum 2013). A mean surface temperature $T=$ $300 \mathrm{~K}$ is assumed in our computation. The other parameters are taken from (Gundlach \& Blum 2013). Based on the thermal inertia $\Gamma=240 \mathrm{Jm}^{-2} \mathrm{~s}^{-0.5} \mathrm{~K}^{-1}$, the grain radius is likely to be in the range $2 \sim 5 \mathrm{~mm}$. In addition, the grain radius may be estimated as ranging from 1.3 to $\sim 31 \mathrm{~mm}$ considering $1 \sigma$ range of thermal inertia. On the basis of this estimation of grain radius, we infer that boulders or rocks may be few on the surface of Bennu, suggesting that the Touch-And-Go Sample Acquisition Mechanism (TAGSAM) designed by the OSIRIS-REx team should find an accessible environment to operate successfully.

\section{Acknowledgments}

This work is financially supported by National Natural Science Foundation of China (Grants No. 11273068, 11473073, 11403105), the Strategic Priority Research ProgramThe Emergence of Cosmological Structures of the Chinese Academy of Sciences (Grant No. XDB09000000), the innovative and interdisciplinary program by CAS (Grant No. KJZD-EW-Z001), the Natural Science Foundation of Jiangsu Province (Grant No. BK 20141509), and the Foundation of Minor Planets of Purple Mountain Observatory. 


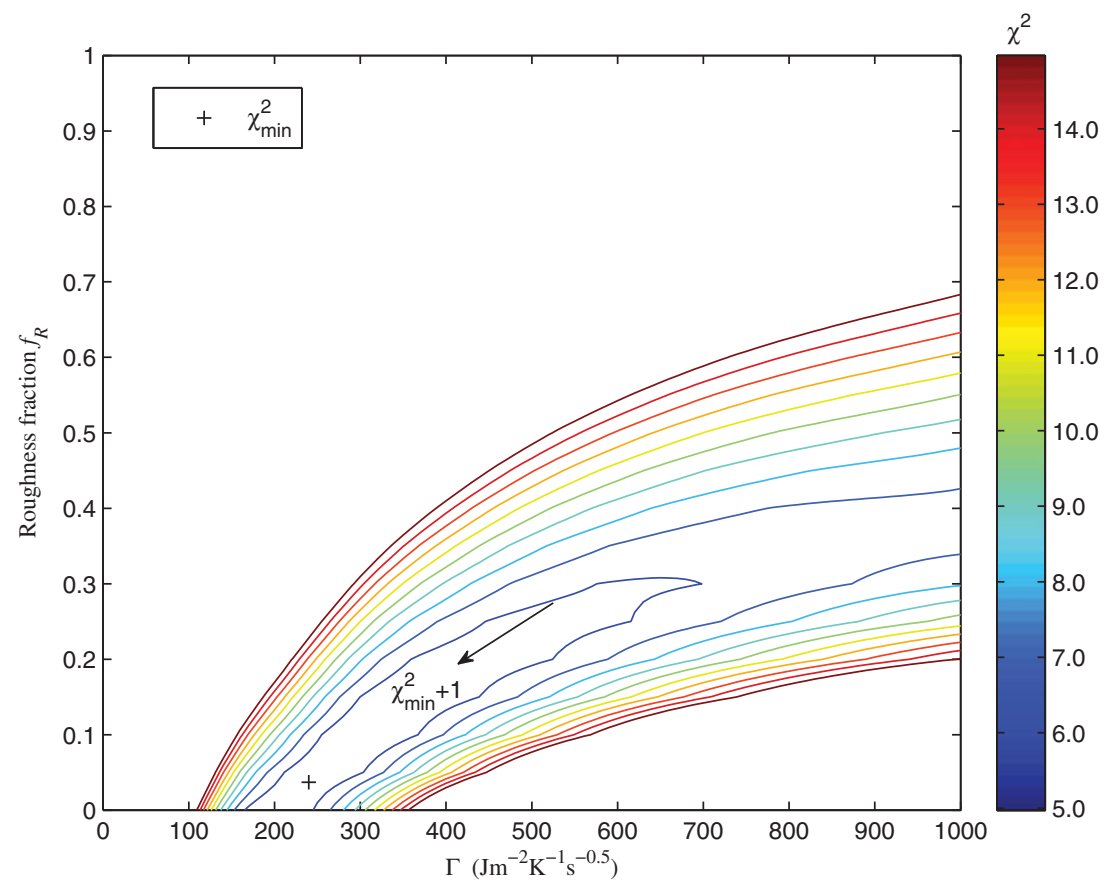

Figure 1. $\chi^{2}\left(\Gamma, f_{\mathrm{R}}\right)$ contour according to ATPM results. The color (from blue to red) relates to the increase of profile of $\chi^{2}$. The blue curve labeled by $\chi_{\min }^{2}+1$ is taken as the $1 \sigma$ limit to the free fit parameters (Emery et al., 2014,Bevington \& Robingson 2003 ).

\section{References}

Bevington, P. R. \& Robinson, D. K. 2003, third ed, Data Reduction and Error Analysis for the Physical Sciences (McGraw-Hill, New York), pp. 194-217

Chesley, S. R., Farnocchia, D., Nolan, M. C., et al. 2014, Icarus, 235, 5-22

Emery, J. P., Fernández, Y. R., Kelley, M. S. P.., et al. 2014, Icarus, 234, 17-35

Gundlach, B. \& Blum, J. 2013, Icarus, 223, 479-492

Lauretta, D. S., and the OSIRIS-REx Team 2012, LPS XXXXIII, Abstract 2491

Milani, A., Chesley, S. R., Sansaturio, M. E., et al. 2009, Icarus, 203(2), 460-471

Müller, T. G., O'Rourke, L., Barucci, A. M., et al. 2012, A $6 A$, 548, A36

Nolan, M. C., Magri, C., Howell, E. S., et al. 2013, Icarus, 226, 629-640

Rozitis, B. \& Green, S. F. 2011, MNRAS, 415, 2042

Yu, L. L., Ji, J. H., \& Wang, S. 2014, MNRAS, 439, 3357

Yu, L. L. \& Ji, J. H. 2015, MNRAS, 452, 368 